\title{
Gravitational wave astronomy with the SKA
}

\author{
G. H. Janssen*1, G. Hobbs ${ }^{2}$, M. McLaughlin ${ }^{3}$, C. G. Bassa ${ }^{1}$, A. T. Deller ${ }^{1}$, M. Kramer ${ }^{4,5}$, \\ K. J. Lee ${ }^{6,4}$, C. M. F. Mingarellii, ${ }^{4,8}$, P. A. Rosado ${ }^{9,10}$, S. Sanidas ${ }^{5}$, A. Sesana ${ }^{11}$, \\ L. Shao $^{12}$, I. H. Stairs ${ }^{13}$, B. W. Stappers ${ }^{5}$, J. P. W. Verbiest ${ }^{14,4}$ \\ ${ }^{1}$ Netherlands Institute for Radio Astronomy (ASTRON), The Netherlands; E-mail: \\ janssen@astron.n 1; ${ }^{2}$ CSIRO Astronomy and Space Science, Australia; ${ }^{3}$ Department of \\ Physics, West Virginia University, USA; ${ }^{4}$ Max-Planck-Institut für Radioastronomie, Bonn, \\ Germany; ${ }^{5}$ University of Manchester, UK, ${ }^{6}$ Kavli Institute for Astronomy and Astrophysics, \\ Peking University, P.R. China; ${ }^{7}$ Theoretical Astrophysics, California Institute of Technology, \\ USA; ${ }^{8}$ School of Physics and Astronomy, University of Birmingham, UK; ${ }^{9}$ Max-Planck-Institut \\ für Gravitationsphysik, AEI Hanover, Germany; ${ }^{10}$ Swinburne University of Technology, \\ Australia ${ }^{11}$ Max-Planck-Institut für Gravitationsphysik, AEI Golm, Germany; ${ }^{12}$ School of \\ Physics, Peking University, P.R. China; ${ }^{13}$ University of British Columbia, Canada; ${ }^{14}$ Universität \\ Bielefeld, Germany
}

On a time scale of years to decades, gravitational wave (GW) astronomy will become a reality. Low frequency $\left(\sim 10^{-9} \mathrm{~Hz}\right) \mathrm{GWs}$ are detectable through long-term timing observations of the most stable pulsars. Radio observatories worldwide are currently carrying out observing programmes to detect GWs, with data sets being shared through the International Pulsar Timing Array project. One of the most likely sources of low frequency GWs are supermassive black hole binaries (SMBHBs), detectable as a background due to a large number of binaries, or as continuous or burst emission from individual sources. No GW signal has yet been detected, but stringent constraints are already being placed on galaxy evolution models. The SKA will bring this research to fruition.

In this chapter, we describe how timing observations using SKA1 will contribute to detecting GWs, or can confirm a detection if a first signal already has been identified when SKA1 commences observations. We describe how SKA observations will identify the source(s) of a GW signal, search for anisotropies in the background, improve models of galaxy evolution, test theories of gravity, and characterise the early inspiral phase of a SMBHB system.

We describe the impact of the large number of millisecond pulsars to be discovered by the SKA; and the observing cadence, observation durations, and instrumentation required to reach the necessary sensitivity. We describe the noise processes that will influence the achievable precision with the SKA. We assume a long-term timing programme using the SKA1-MID array and consider the implications of modifications to the current design. We describe the possible benefits from observations using SKA1-LOW. Finally, we describe GW detection prospects with SKA1 and SKA2, and end with a description of the expectations of GW astronomy.

Advancing Astrophysics with the Square Kilometre Array

June 8-13, 2014

Giardini Naxos, Italy

${ }^{*}$ Speaker. 


\section{Introduction}

Radio observations of binary pulsars provide irrefutable evidence that gravitational waves (GWs) exist. The impact of their emission on e.g. the orbital period of binary pulsars can be used to test general relativity and other theories of gravity (e.g. Taylor \& Weisberg 1982; Kramer et al. 2004; Cordes et al. 2004). The effect of external GWs on pulsar timing (Detweiler 1979) can be used to make a direct detection of low-frequency GWs through the analysis of a large ensemble of pulsars, forming a galactic-scale GW detector: a Pulsar Timing Array (PTA; Hellings \& Downs 1983; Romani 1989; Foster \& Backer 1990). Global efforts are underway to directly detect low-frequency GWs via PTA experiments.

In this chapter we describe how a PTA project for the SKA will allow the characterisation of GW signals. PTA projects are based on pulsar timing observations in which pulse times of arrival (ToAs) for a sample of pulsars are measured. The ToAs are subsequently compared with predictions for those arrival times based on simple physical models of the pulsars. Any differences between the predicted and measured ToAs are referred to as the "timing residuals" and indicate that a physical phenomenon, not (or incorrectly) included in the model, is affecting the pulse ToAs at a measurable level. Residuals caused by the irregular rotation of a given pulsar, variability in the interstellar medium, or inaccuracies in the timing model parameters are typically different between pulsars. In contrast, GWs passing the Earth will lead to timing residuals whose functional form will depend upon the pulsar-Earth-GW angle. PTA projects therefore differ from more traditional pulsar timing experiments in that they aim to extract common signals present within the timing residuals for multiple pulsars in order to detect GWs and other correlated signals, like fluctuations in global atomic time standards (Hobbs et al. 2012) or unmodelled effects in the Solar system ephemeris that is used to convert pulse ToAs to the Solar system barycentre (Champion et al. 2010).

Long-term observations of millisecond pulsars were carried out at telescopes around the world starting in the 1980s and 1990s, producing data sets that can be usefully added to modern PTA observations (see e.g. Kaspi et al. 1994). Three PTAs are presently in existence. The European Pulsar Timing Array (EPTA; see Kramer \& Champion 2013, for a review) uses five radio telescopes in Europe (the 100-m Effelsberg telescope in Germany, the 76-m Lovell telescope at Jodrell Bank in the UK, the 94-m equivalent Nançay telescope in France, the 64-m Sardinia radio telescope in Italy, the 94-m equivalent Westerbork Synthesis Radio Telescope in The Netherlands) and complementary low-frequency $(<250 \mathrm{MHz})$ observations obtained with LOFAR (van Haarlem et al. 2013; Stappers et al. 2011) for some pulsars. The North American Nanohertz Observatory for Gravitational Waves (NANOGrav; Demorest et al. 2013), uses the 305-m Arecibo and 100-m Green Bank telescopes, and the Parkes Pulsar Timing Array (PPTA; Manchester et al. 2013), uses the 64-m Parkes telescope. All collaborations have well established pulsar timing programmes on at least 20 millisecond pulsars with time baselines of 10 years or more. Regular timing observations for these pulsars are being obtained over a wide range of observing frequencies (300 to $3000 \mathrm{MHz}$ ) to mitigate the effects of variations in dispersion. Since 2008, the three PTAs are collaborating as members of the International Pulsar Timing Array (IPTA; Manchester \& IPTA 2013). Over the years, all PTAs have increased their sensitivity to GWs through improvements in instrumentation, software, and observing cadence and data span. Furthermore, the EPTA is using interferometric techniques to form the LEAP telescope, a tied-array of the five large European radio telescopes 
(Kramer \& Stappers 2010; Bassa et al. in prep.). In the near future it is expected that PTA observations will be also carried out using the SKA Pathfinder telescope MeerKAT, and with the Five-hundred-meter Aperture Spherical Telescope (FAST; Hobbs et al. 2014).

There is a broad GW spectrum, rich with information ranging from processes in the early Universe to compact binary coalescences.

Cosmological GW signatures at frequencies of $\sim 10^{-16} \mathrm{~Hz}$ are targeted by cosmic microwave background (CMB) polarisation experiments such as BICEP2 and Planck (e.g. Ade et al. 2014; Planck Collaboration 2014). Ground-based interferometer GW observatories, like the (advanced) LIGO and Virgo, and a space-based mission, like the proposed eLISA, operate at $\sim 1-10^{3} \mathrm{~Hz}$ and $\sim 10^{-4}-1 \mathrm{~Hz}$, respectively (see e.g. Pitkin et al. 2011; eLisa Consortium 2013, for overviews). Therefore, PTAs with sensitivities peaking at frequencies of $10^{-9}-10^{-7} \mathrm{~Hz}$ represent a unique complementary laboratory to other GW experiments and are able to detect and study unique classes of sources (see Figure 1).

Throughout this chapter we concentrate on the detection and subsequent analysis of GWs. However, the data sets produced for this work will be useful in numerous ways; including studying the interstellar medium, the pulsars themselves, terrestrial time standards, planetary ephemerides, the solar wind, and testing theories of gravity. We first describe the expected sources of GW emission detectable by PTAs ( $\$ 2$ ). In $\S 3$ we describe how the SKA will enable both GW detection and GW source characterisation. In $\S 4$ we highlight various limiting noise processes that need to be accounted for in the detection analyses. In $\$ 5$ we describe a realistic PTA for the SKA which includes a plausible observing strategy. The effect of a cut in the baseline design is described in $\S 5.4$. We describe the future of GW astronomy with the SKA in $\S 6$.

\section{Sources of gravitational waves}

There are numerous GW sources that could lead to detectable signatures in pulsar data sets. Most recent work has concentrated upon searching for a GW background formed by the superposition of a large number of GWs from supermassive black hole binaries (SMBHBs; see e.g. Sesana et al. 2008; Ravi et al. 2012; Ölmez et al. 2010), cosmic strings (e.g. Sanidas et al. 2012) or inflation (e.g. Tong et al. 2014). Various algorithms have been published to constrain such a background or to attempt to detect the background (see e.g. Jenet et al. 2006; Yardley et al. 2011; van Haasteren et al. 2011; Sanidas et al. 2012; Demorest et al. 2013; Shannon et al. 2013). These bounds have assumed that the GW background spectrum is a power law defined by an amplitude $A$ and spectral index $\alpha$. The stochastic GW backgrounds of the sources probed by PTAs have a range of spectral indices, depending on the details of the theoretical models, with the most widely used values being $\alpha \sim-2 / 3$ for SMBHBs without environment interactions (Phinney 2001), $\alpha \sim-7 / 6$ for high emission modes from cosmic string cusps and $\alpha \sim-1$ for slow-roll inflation. Current bounds are beginning to rule out some extreme models for the coalescence rate of SMBHBs and for the tension and loop size of cosmic strings. The most stringent published limit, from PPTA data, on characteristic amplitude due to a stochastic GW background is $2.4 \times 10^{-15}$ (at a reference frequency of $\mathrm{yr}^{-1}$; Shannon et al. 2013). Figure 1 shows a nominal upper bound at the level we expect to reach with the full IPTA $\left(h_{c} \sim 4 \times 10^{-15}\right.$ at $5 \times 10^{-9} \mathrm{~Hz}$, which corresponds to about $h_{c} \sim 10^{-15}$ at a frequency 


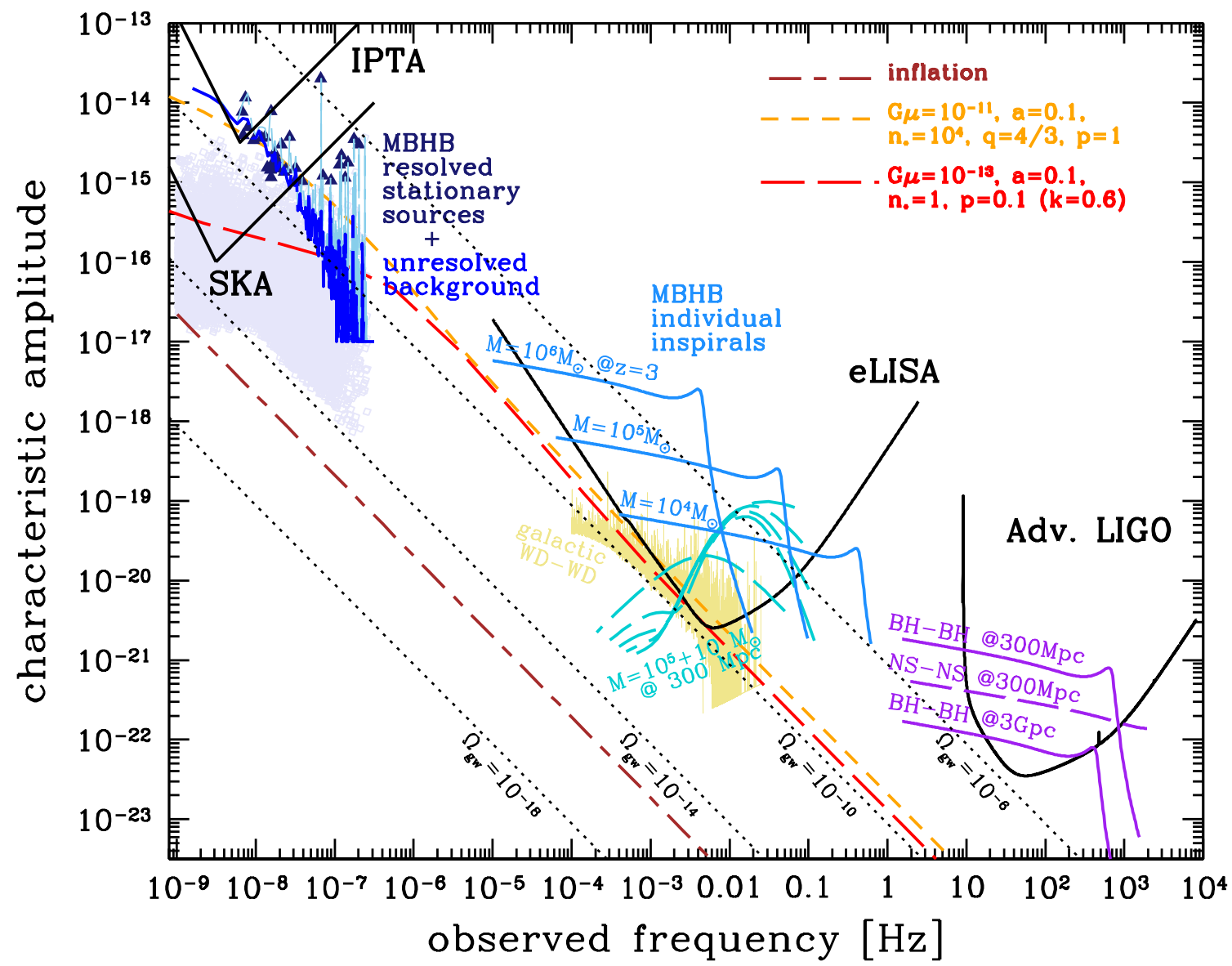

Figure 1: The gravitational wave landscape: characteristic amplitude $\left(h_{c}\right)$, vs frequency. In the $\mathrm{nHz}$ frequency range a selected realisation of the expected GW signal from the cosmological population of SMBHBs is shown. Small lavender squares are individual SMBHB contributions to the signal, the dark blue triangles are loud, individually resolvable systems and the blue jagged line is the level of the unresolved background. Nominal sensitivity levels for the IPTA and SKA are also shown. In the $\mathrm{mHz}$ frequency range, the eLISA sensitivity curve is shown together with typical circular SMBHB inspirals at $z=3$ (pale blue), the overall signal from Galactic WD-WD binaries (yellow) and an example of extreme mass ratio inspiral (aquamarine, only the first 5 harmonics are shown). In the $\mathrm{kHz}$ range an advanced LIGO curve (based on calculations for a single interferometer) is shown together with selected compact object inspirals (purple). The brown, red and orange lines running through the whole frequency range are expected cosmological backgrounds from standard inflation and selected string models, as labeled in figure. Black dotted lines mark different levels of GW energy density content as a function of frequency $\left(\Omega_{g w} \propto h_{c}^{2} f^{2}\right)$.

of $\mathrm{yr}^{-1}$ for an extrapolated $f^{-2 / 3}$ power law), and the expected levels to be reached by the SKA $\left(h_{c} \sim 10^{-16}-10^{-17}\right.$ at a reference frequency of $\left.\mathrm{yr}^{-1}\right)$.

Pulsar timing can also be used to search for individual sources of GWs: individual SMBHBs that produce a continuous wave. A single (effectively non-evolving) circular binary system of supermassive black holes produces a sinusoidal signal. Various algorithms have recently been developed to search for such waves. These include a frequentist-based approach (Jenet et al. 2004; Yardley et al. 2010) and Bayesian methods (van Haasteren et al. 2009; Lee et al. 2011; Babak \& 
Sesana 2012; Ellis et al. 2012; Petiteau et al. 2013; Ellis 2013; Arzoumanian et al. 2014). Pulsar timing experiments are sensitive to sources emitting GWs with periods from a few weeks (defined by the average observing cadence in the PTA) to around the total duration of the data set. Using the algorithms mentioned above, bounds have been placed on such GWs in this frequency range. For existing PTAs, GWs with periods of one or half a year are undetectable because of the necessity of fitting for each pulsar's position and parallax during the analysis (this can be improved by using astrometric parameters independently measured through VLBI observations; see §3.4). Figure 1 shows examples of individually detectable SMBHB systems in a selected synthetic realisation of the cosmic SMBHB population using dark-blue triangle symbols. Another source of GWs in the PTA band is caused by the merger of two supermassive black holes; this may lead to a permanent deformation of their surrounding space-time, which can be detectable as a memory event that takes the form of a "glitch" in the timing residuals (van Haasteren \& Levin 2010; Cordes \& Jenet 2012).

\section{Detecting gravitational waves}

Direct gravitational wave detection and its subsequent confirmation is likely to be a long process. Currently, searches for GWs with PTAs are setting evermore stringent upper limits on the amplitude of the stochastic GW background. At some time (our predictions suggest that this will be soon), the signatures of the GWs (most likely red noise from a GW background) will become apparent in the timing residuals of the pulsars with the lowest rms timing residuals. At this point the bounds will not improve with time and it will become possible to study the signal, determine its amplitude, frequency (for a continuous wave source) or spectral index (for a background). However, confirmation of the GW nature of the signal will only come when the expected correlation signature for an isotropic stochastic GW background (Hellings \& Downs curve; 1983), is found in the timing residuals of many pulsars. Making a highly significant measurement of such correlations requires much longer data sets than is needed for a bound. We therefore consider the following three scenarios for the SKA PTA project: 1) no sign of a GW signal has been seen in the existing PTA data, 2) a red noise signal has been identified, but the data sets are insufficient to "make a detection" and 3) a GW detection has already been made. We conclude this section by highlighting the role of Very Long Baseline Interferometry for improving the SKA's sensitivity to GWs.

\subsection{No signal has been seen}

With the current limits (e.g. Shannon et al. 2013) starting to constrain the low-redshift SMBHB population, it is likely that, by the time the SKA1 is commissioned, data from existing PTA experiments will already indicate the presence of a GW signal. However, if no such signal is observed then it is likely that either the pulse arrival times have not been measured with sufficient precision, that jitter noise is dominating the data sets, that not enough pulsars are being observed or that timing noise is affecting the detectability of the signal. The SKA can provide significant improvements in all these areas. With its unprecedented sensitivity the SKA should be able to observe a large number of currently unusable millisecond pulsars. The timing precision achievable for many pulsars will be limited by fluctuations in the pulse shape from individual pulses. This "jitter noise" (see $\S 4$ for more details) can be mitigated using the SKA by forming sub-arrays, and thereby integrating 
for longer on multiple pulsars in parallel. With a much larger number of pulsars, timed with better precision than currently available, it is likely that GW detection can be made with shorter data spans (Rosado, Sesana \& Gair in prep.). Having a shorter time span may also mitigate issues relating to timing noise. Moreover, we will be able to combine new data with archival data collected up to SKA operation, so that the crucial time baseline can be extended, albeit with a lower sensitivity.

\subsection{Red noise signal has already been identified}

If red noise (or a low-frequency signal) has been identified then the SKA data sets can be used in conjunction with the longer existing data sets to improve their sensitivity to GWs. Research is ongoing to make reasonable predictions for GW detection for a specified real (or future) data set. Siemens et al. (2013) made an initial attempt to calculate the scaling of the signal-to-noise of an idealised PTAs as the signal builds up in the array. They demonstrate that the significance of a detection of a GW signal will increase with longer data spans at a different rate for these three different regimes: 1) where the GW signal is weak and the white noise dominates, 2) where the GW signal is strong and 3) an intermediate regime where only the power in the lowest frequencies of the $\mathrm{GW}$ background is above the white noise level. A realistic array is likely to be complex and contain some pulsars for which the GW signal is weak compared to other red noise signals in the data, and other pulsars for which the signal will be comparatively strong. Consequently, it is challenging to make analytic predictions for the time to detection for a given array. A full analysis will require an improved understanding of the expected signal strength and the noise processes (such as timing noise and jitter noise), as well as a model-independent characterisation of the statistical properties of the signal.

\subsection{GW detection has already been made}

If a GW signal has already been detected and published then the SKA would begin the exciting work of studying the properties of the GWs and characterising the GW background and sources (see also §6). For instance an SKA PTA would:

- Confirm the detection: When a stochastic GW background has been detected, the signficance of the detection will increase as the quality and durations of data sets increase. SKA data sets would provide dramatically increased sensitivity and could be used to provide a confirmation of the detection, with a shorter timespan of data, different instrumentation, and different sample of millisecond pulsars.

- Confirm the nature of the GW signal: The induced residuals from a background of cosmic strings, the inflationary era, and merging supermassive black holes will all have the same characteristic angular signature. Futhermore, they all have red power spectra which will initially be indistinguishable. The SKA should be able to distinguish between the different spectral indices for the various sources. A confirmed GW background from supermassive black hole mergers would provide strong evidence of the existence for a large population of SMBHBs where each binary is separated by less than a parsec, proving another crucial prediction of the hierarchical model of structure formation (Sesana 2013b). Moreover, Mingarelli et al. (2013) show that some level of anisotropy may be present in the stochastic GW 
background, and characterisation of the GW energy density at different angular scales carries information on the nature of the GW background. With increasing spatial and frequency resolution, SKA will be also able to identify the loudest astrophysical GW sources, disentangling individual SMBHBs from the overall GW background (e.g. Babak \& Sesana 2012; Petiteau et al. 2013)

- Detect a turn-over in the GW spectrum: For a background of SMBHBs, the expected simple power-law form for the characteristic strain spectrum is only valid for circular binaries whose evolution is driven solely by GW emission (Sesana 2013a; Ravi et al. 2014). Any spectral turn-over would indicate interactions between the supermassive black holes and their environment (for instance, the effects of stellar scattering the presence of circumbinary discs). Furthermore, the GW spectrum for a background formed from cosmic strings could also have a complicated shape in the region of interest for PTA experiments (Sanidas et al. 2013).

\subsection{The role of VLBI}

For each pulsar in a PTA, the effect of a gravitational wave can be partially absorbed into parameters in the pulsar timing model, reducing low-frequency sensitivity. It is therefore advantageous to obtain independent measurements of the parameters that can be held fixed in the pulsar timing model. The most common method of providing external information is via Very Long Baseline Interferometry (VLBI), which yields very accurate positions in the International Celestial Reference Frame (ICRF). By repeating such measurements over time, pulsar reference positions, proper motions, parallaxes, and in some cases orbital parameters are obtained (e.g. Deller et al. 2013). In addition to being an independent source of information, unaffected by low-frequency GWs, VLBI astronomy gives higher precision than is possible via timing in almost all cases (see Paragi et al. 2015, for more details). A caveat is that at present transferral from the ICRF to the Solar system barycentric frame reduces the usefulness of the VLBI-derived reference position values, as the uncertainty in the frame tie (presently $\sim$ milliarcseconds) is orders of magnitude larger than the formal uncertainty (but see however the third point below).

The three main benefits of VLBI information for gravitational wave searches are as follows:

1. The sensitivity is greatly improved to GWs with periods of $\sim 0.5$ years and $\sim 1.0$ years, due to the GW contribution no longer being absorbed into the fit for parallax and proper motion (plus reference position if transferrable; e.g. Madison et al. 2013).

2. If very accurate distances can be derived for one or more pulsars, such that the remaining uncertainty is a small fraction of a gravitational wavelength, then sensitivity to individual sources can be greatly enhanced. In this case, the contribution to the timing residuals from the gravitational wave at the pulsar (the "pulsar term") can be coherently accounted for (Lee et al. 2011), which is a particular benefit for the pulsars with the highest timing precision. If several such measurements can be made and multiple individual gravitational wave sources can be detected, then it will even be possible to determine the distances of other pulsars in the array to high precision using the gravitational wave fit directly (Lee et al. 2011; Boyle \& Pen 2012) 
3. By comparing a large sample of VLBI positions and pulsar timing positions, it will be possible to improve the planetary ephemeris used to convert pulsar arrival times to the solar system barycentre, which will lead to improved accuracy for timing-derived pulsar positions (Madison et al. 2013).

\section{Limiting noise processes}

The raw sensitivity of the SKA will enable ToAs to be determined with much higher precision and will allow a much larger sample of pulsars to be included in PTA experiments. However, even though the ToA timing precision will increase, a limit may exist in timing precision due to various effects like pulse jitter on short time scales, intrinsic pulsar timing noise on longer time scales and interstellar medium effects such as scattering (Cordes \& Shannon 2012). In this section we describe the various noise processes that, if uncorrectable, will limit the achievable precision with the SKA.

\subsection{Pulse jitter}

The phenomenon of "jitter noise" results from intrinsic variability in the shape of individual pulses from a pulsar. This has been shown to be the limiting factor in the achievable timing precision for PSR J0437-4715 (Osłowski et al. 2011), where a floor of the timing precision of $\sim 40 \mathrm{~ns}$ in a one-hour observation (and worse with shorter observations) is reached, even when using the largest telescopes available. Similar results have been found for PSR J1713+0747 using Arecibo observations (Shannon \& Cordes 2012).

Jitter noise is thought to be, at some level, a limiting noise process. However, an improvement of nearly $40 \%$ in the rms timing residual for PSR J0437-4715 was achieved by using information from the polarised pulse profile (Osłowski et al. 2013), with the result ultimately limited by variable Faraday rotation in the Earth's ionosphere. Current studies of jitter noise are limited by the sensitivity of available telescopes, with only a handful of millisecond pulsars having detectable single pulses. As telescopes become larger, we will be able to significantly improve our understanding of the jitter phenomenon. This, along with robust calibration of data, could possibly lead to the development of strategies to remove the effects of jitter from SKA data.

If jitter noise is uncorrectable, the SKA holds no advantage for a jitter-dominated pulsar over a less-sensitive telescope. It is, however, likely that the SKA will observe many pulsars that are in the jitter-dominated regime. In order to maximise the scientific output it will therefore be essential to sub-array the telescope, enabling multiple pulsars to be observed simultaneously for long sessions.

\subsection{Timing noise}

An analysis of timing irregularities for 366 pulsars allowed the first large-scale analysis of timing noise over time-scales of $>10 \mathrm{yr}$ (Hobbs et al. 2010). The youngest pulsars were shown to be dominated by the recovery from glitch events (sudden increases in the spin frequency), while the timing irregularities for older pulsars seemed to exhibit quasi-periodic structure. Millisecond pulsars were included in the sample, but the data set used (from the Lovell telescope at Jodrell Bank) did not allow an analysis of timing noise with the precision necessary for PTA-research. The first major assessment of timing noise on the precision timing of millisecond pulsars concluded that 
timing noise is present in most millisecond pulsars and will be measurable in many objects when observed over long data spans (Shannon \& Cordes 2010).

There is currently no proven method for removing timing noise. Furthermore, the power spectrum of timing noise is red, with most of the power at low frequencies, and for some MSPs can be even steeper than the expected spectrum of the stochastic GW background. Timing noise will therefore provide a stringent limit for long-term timing projects. The SKA will need to either observe pulsars that exhibit small amounts of timing noise, observe a sufficiently large number of pulsars to achieve the science goals before timing noise dominates the residuals, or observe over long enough data spans for the timing noise level to plateau.

For young pulsars, timing noise can be modelled as a process in which the spin-down rate of the pulsar flips between two stable states (Lyne et al. 2010). These variations are accompanied by pulse profile changes as well. This leads to the possibilities that 1) millisecond pulsar timing noise can also be modelled as a two-state process and that 2) the state at a given time can be determined from the pulse shape. If true, it may be possible to completely remove the effect of timing noise.

\subsection{Effects from the interstellar medium}

The free electron content of the Galaxy affects radio wave propagation in a number of different ways. Most importantly, the presence of these charged particles changes the refractive index of the interstellar medium, and therefore the group velocity of the radio waves. In the context of pulsar timing, this is observed as a dispersion delay that strongly scales with the observing frequency $\left(\tau_{D M} \propto v^{-2}\right)$ and which is characterised by the dispersion measure (DM), defined as the integrated electron density between the pulsar and Earth, in units of $\mathrm{cm}^{-3} \mathrm{pc}$ (Lorimer \& Kramer 2005).

This dispersive delay in itself does not affect high-precision pulsar timing experiments, but because pulsars are high-velocity objects (Hobbs et al. 2004), the integrated electron density between Earth and a given pulsar changes slightly as the line of sight samples different regions of the turbulent interstellar medium (ISM) (Brisken et al. 2010). This makes the DM and its associated frequency-dependent delay time-variable, which in turn introduces unmodelled variations in the pulsar timing data. This time variability is the main cause of unmodelled ToA variations in high-precision pulsar timing data (Verbiest et al. 2009); therefore, mitigating this effect is a critical challenge in moving towards gravitational-wave detection (Keith et al. 2013; Lee et al. 2014).

There are essentially three approaches to dealing with this problem; and most likely a combination of approaches will be needed in order to obtain the best results.

Firstly, because the dispersive ISM delay is strongly frequency-dependent, observations with large bandwidths can measure these delays with very high accuracy. Lee et al. (2014) have evaluated the covariance of DM variation and timing precision, where they found that a wider band and better precision is necessary to reduce such covariance. Several pathfinder projects where ultrabroadband receivers simultaneously observe at all frequencies between $\sim 500 \mathrm{MHz}$ and $\sim 2 \mathrm{GHz}$ are ongoing, and the efficacy of this approach is being evaluated. This method is unique because the data used for the high-precision timing project are the same data used for DM determination, allowing a simultaneous determination of all parameters at the cost of increased covariance between parameters. The main challenge of this method involves possible corruptions introduced by unmodelled frequency-dependence of the pulse profile and possible frequency-dependent behaviour of pulse phase jitter (see §4.1). In recent work by Liu et al. (2014) and Pennucci et al. (2014) 
algorithms have been presented to model the frequency dependence of the pulse profile and improve the timing accuracy of pulsar observations obtained with wide band instruments. However, to accurately asses these problems SKA1 sensitivity is required.

A second approach is, instead of determining the DM values simultaneously across a very wide bandwidth, to use only the very lowest frequencies available $(\sim 100-300 \mathrm{MHz})$ to determine the DM values independently from the timing data obtained at higher frequencies. This approach puts smaller demands on available observing time and does not require a very high instantaneous bandwidth. One potential problem with this approach, however, is that the lowest observing frequencies may actually sample a different part of the ISM than the higher observing frequencies, because the refractive angles are different for different frequency bands. In practice this effect should be limited for a homogeneous and smooth ISM, but the turbulent ISM can hardly be expected to be smooth and homogeneous. This approach is currently being tested with the LOFAR pathfinder and as yet the inhomogeneities of the ISM do not seem to pose a problem (Hassall et al. 2012).

Finally, with sufficient sensitivity, a large telescope could collect pulsar timing data at high observing frequencies ( $3 \mathrm{GHz}$ and up). This would strongly diminish the effects of DM variations and thereby avoid the need to correct for variable interstellar delays. The steep spectral index of most pulsars will require a longer integration time at these frequencies, but this may be required to combat the effects of pulse phase jitter. The clear disadvantage of this method, is two-fold: most importantly, without corrections for variable DMs, the red noise spectrum of the DM variations (Armstrong et al. 1995) will bring these variable delays to a level where they do affect the timing and hence gravitational-wave sensitivity. At that point, if no other multi-frequency information is available the data set will no longer be useful, as back-correction for DM variability is not possible. A secondary draw-back is that the spin-off science of studying the hot ionised component of the ISM would become impossible with high-frequency data alone.

A more subtle effect of the ionised interstellar medium is interstellar scattering. This effect is primarily observable through relatively innocuous variations in the pulsar's brightness (scintillation), caused through interference between rays with slightly different path lengths. In a more extreme form, this difference in path length between rays that are scattered off different density inhomogeneities in the ISM actually causes significant amounts of flux to arrive at the telescope with an observable delay. This causes the shape of the pulse profile to display an exponential decay (a so-called "scattering tail"). This scattering degrades timing precision because the scattering tail washes out any fine-scale features of the pulse profile (which are particularly useful in locking down the phase of the observed pulses). More importantly, if the scattering is time-variable (and, again, given the pulsar's high spatial velocity and the turbulent character of the ISM, this is to be expected), then the shape of the pulse profile will vary in time, affecting the timing in an unpredictable manner. Because of the strong dependence of scattering on density inhomogeneities, the scaling of scattering with frequency is less well-defined than for dispersive delays, but scattering strength typically scales even more strongly with frequency ( $\propto v^{-4.4}$, Bhat et al. 2004), providing another impetus for high-frequency timing observations. While it is impossible to remove scattering exactly as in the case of dispersive delays, one promising technique termed cyclic spectoscopy has been demonstrated to retrieve the intrinsic, unscattered pulse profiles for one highly scattered, bright millisecond pulsar (Demorest 2011; Walker et al. 2013). A wider study is required to assess how broadly this method will be applicable to less scattered and weaker PTA pulsars. 


\section{A realistic PTA using the SKA}

In this section we consider a realistic PTA project carried out using the SKA. We consider the expected number of pulsars, the plausible timing precision achievable, the time span for the experiment, specific issues relating to the SKA design, and the other telescopes available for PTA observations that will be operating at the same time as the SKA1.

\subsection{Telescopes observing pulsars in the SKA1 era}

While the SKA1 is being commissioned, the existing radio telescopes are expected to continue their PTA observations. Even with the increased sensitivity that the SKA1 provides, combining SKA1 data with the long time baselines of the existing PTAs improves the sensitivity for the detection of gravitational waves. Furthermore, it is essential for long-term pulsar timing experiments that a long overlap period is included between two observing systems in order to characterise the timing offsets between them. Finally, obtaining PTA observations with multiple telescopes provides greater cadence and coverage of observing frequencies.

As the SKA1 is commissioned it is likely that most of the current 100-m class telescopes will still be observing pulsars along with the Five-hundred-meter Aperture Spherical Telescope (FAST) and the Xinjiang Qitai 110m Radio Telescope (QTT) in China. The Giant Metrewave Radio Telescope (GMRT) in India may begin to play a larger role, as its capability to simultaneously observe at 300 and $600 \mathrm{MHz}$ is useful for DM corrections. LOFAR could also be useful for DM montoring of Northern pulsars. The Murchison Widefield Array will complement LOFAR observations in the Southern hemisphere and can be used to monitor effects of the interstellar medium. In addition, the upcoming 100-m class CHIME (Canadian Hydrogen Intensity Mapping Experiment) will make daily observations of Northern-sky pulsars at frequencies between 400 and $800 \mathrm{MHz}$, providing densely-sampled records of DM and scattering changes toward the relevant MSPs.

At the time that SKA1 is commissioned the ASKAP COAST project is likely to be underway. This project aims to discover new pulsars (many of which may become part of the SKA PTA sample) and to carry out timing observations. The ASKAP sensitivity is relatively low and therefore it is likely that the majority of high precision timing being carried out in the Southern hemisphere at the time will be being obtained with Parkes telescope and the MeerKAT array. The baseline design indicates that the MeerKAT array will be combined as part of the SKA1-MID. However, it will be essential either for the Parkes telescope to provide an overlap period with the SKA1, or for MeerKAT to continue to operate in an overlap mode with the SKA1-MID for at least 1 year.

With the collecting area of FAST and the SKA1-MID, it is likely that most of the currently observed millisecond pulsars will be jitter dominated. An analysis of a PTA for the FAST telescope (Hobbs et al. 2014) showed that 1) long observation times $\sim 1$ hour per pulsar will still be required to obtain high time-precision observations for most of the currently known pulsars and 2) for pulsars that are jitter dominated there is no advantage (for timing purposes) in using a very large telescope (see also Liu et al. 2012). However, the SKA1-MID has the advantage that sub-arrays can be formed enabling observations of multiple pulsars (at, or close to, the jitter level) simultaneously.

Optimising the observation strategy has been investigated by Lee et al. (2012), where both the single and multiple telescope optimisations were considered under the influence of radiometer, jitter, and red noise. They found that, because of jitter noise, it is preferable to use a smaller telescope 


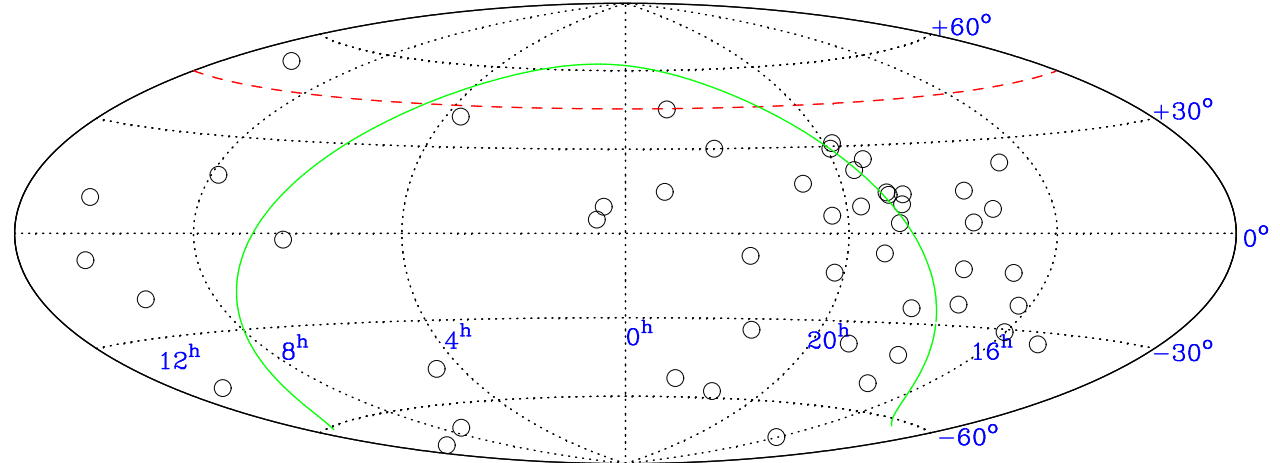

Figure 2: Expected sky coverage of the SKA, overlaid with the positions of the current pulsars being observed as part of the IPTA project (open circles). For an array mainly at latitude $-30^{\circ}$ the telescope will have an approximate declination range from $-90^{\circ}$ to $+45^{\circ}$ (red dashed line). We note that 49 out of the 50 current IPTA pulsars could be observed by the SKA. The green line indicates the Galactic plane.

to observe the brightest pulsars. In this way, for certain bright pulsars, it can be more efficient to split the SKA1-MID (or SKA1-LOW) into sub-arrays or use existing 100-m class telescopes.

\subsection{Observing strategy}

Current predictions for the detectable GW signal suggest that high cadence observing is important, but ensuring a long baseline of observations over years to decades is essential. A realistic scenario would be to observe a sample of pulsars once per week. In order to obtain the highest possible timing precision it will be necessary to obtain high quality polarisation calibration. This will require dedicated pointing directly at the pulsar. It is unlikely that more than one PTA-quality pulsar will be within the primary beam of the telescope, meaning that multiple tied-array beams will not necessarily be useful for the high precision timing programme, however the remaining beams can be used in parallel for other timing projects. The full sensitivity of the telescope will not be required in cases where the pulsars are dominated by jitter noise. In that case, it is more efficient to sub-array the telescope to observe multiple pulsars simultaneously.

It is likely that around 50 pulsars could be observed during a single 24-hour observing block providing a timing precision of $<100 \mathrm{~ns}$. This gives observation times of $\sim 15$ minutes per pulsar (assuming some nominal setup time for each observation) assuming that each pulsar is observed with two receivers. In order to account for DM and scattering variations it will be necessary to observe at a wide frequency range. Because of the steep radio spectra of pulsars, current PTA observations are limited to frequencies below $\sim 3 \mathrm{GHz}$. With SKA1-MID, PTA observations will be carried out with bands 2,3 and $4(0.95$ to $1.76 \mathrm{GHz}, 1.65$ to $3.05 \mathrm{GHz}$ and 2.80 to $5.18 \mathrm{GHz}$ respectively). As discussed in $\$ 4.3$, lower frequency bands, and SKA1-LOW should be used for tracking variations in dispersion measures.

\subsection{Offline data analysis}

The data taken for the current PTA projects are generally stored in greatly compressed form, with typical data file sizes for a single pulsar observation currently around $\sim 1 \mathrm{~GB}$ (for a data file containing 64 subintegrations, 1024 frequency channels and 2048 phase bins). These data 
sizes have not posed any major challenges for current timing programmes. However, as data rates increase, recording, archiving and processing are all becoming more challenging. With wider bands available it is plausible that the SKA would produce data files of $\sim 10 \mathrm{~GB}$ for each standard observation. A year of observing 100 pulsars with weekly cadence would lead to $\sim 60 \mathrm{~TB}$ for each backend instrument. This is not impractical, but it will be necessary to provide copies of these data at the data processing centres. Furthermore, it is likely that new methods for high-precision timing will soon be developed. These could include using bright individual pulses, higher-order moments of the electric field, or dynamic spectra, all of which would require additional processing and the storage of additional products. Some methods, such as cyclic spectroscopy, may even require the storage of all data in raw, baseband form.

The supporting infrastructure that enables multiple copies of the data to be replicated at different sites and enables the processing of that data will need to be planned in detail. Keeping these systems operating and providing data and processing power to the community is non-trivial and will require significant management. Standard processing of the pulsar arrival times requires minimal computing resources. A typical set of 260 observations for a given pulsar spread over five years can easily be processed with the pulsar timing software package TEMPO2 (Hobbs et al. 2006) on a laptop computer. However, with the wide bandwidths available it is likely that a large number of ToAs at different frequencies will be calculated and stored for a single observation. Many of the gravitational wave detection codes (both frequentist and Bayesian based methods) require the analysis of $N_{\text {toa }} \times N_{\text {toa }}$ matrices and many of the algorithms require $N_{\text {toa }}^{3}$ operations. This can quickly become prohibitive for a large number of ToAs, particularly if it is necessary to also run the detection code on a large number of simulated data sets. It is therefore essential that the data sets can be processed on high performance computers. Much of the pulsar processing software is now being modified to allow the use of Graphics Processing Units (GPUs) where available. GPUs are well suited for dealing with processing large matrices and we therefore recommend that the infrastructure created for the SKA allows the data to be processed both on Central Processing Units (CPUs) and also on GPUs.

\subsection{Time to detection}

In general, high-precision pulsar timing experiments rely on a sensitive telescope with sufficient frequency coverage to mitigate noise sources. As mentioned in $\S 3$, the success of detecting and studying GWs with the SKA depends on the quality of the observations and the number of pulsars in the array. In Figure 3, a comparison is shown between different PTA configurations and the probabilities of detecting a $\mathrm{GW}$ background or a continuous $\mathrm{GW}$ source. To produce these plots, $\sim 10^{5}$ simulations of the GW signal from SMBHBs have been analysed (Rosado, Sesana \& Gair in prep.). All the models assumed in the production of these simulations are consistent with current electromagnetic observations (Sesana 2013a).

With the SKA as described in the baseline design document, it will have a 50\% probability of detecting a GW source (either single or background) after five years. This is a conservative estimate since pre-existing IPTA data sets have not been included in the probability calculations. The PTA project on the SKA would not be significantly affected by a modification to the maximum and minimum baselines available. However, significant changes to the GW-sensitivity of the PTA would occur if a major reduction was made to the telescope sensitivity (e.g. fewer dishes 

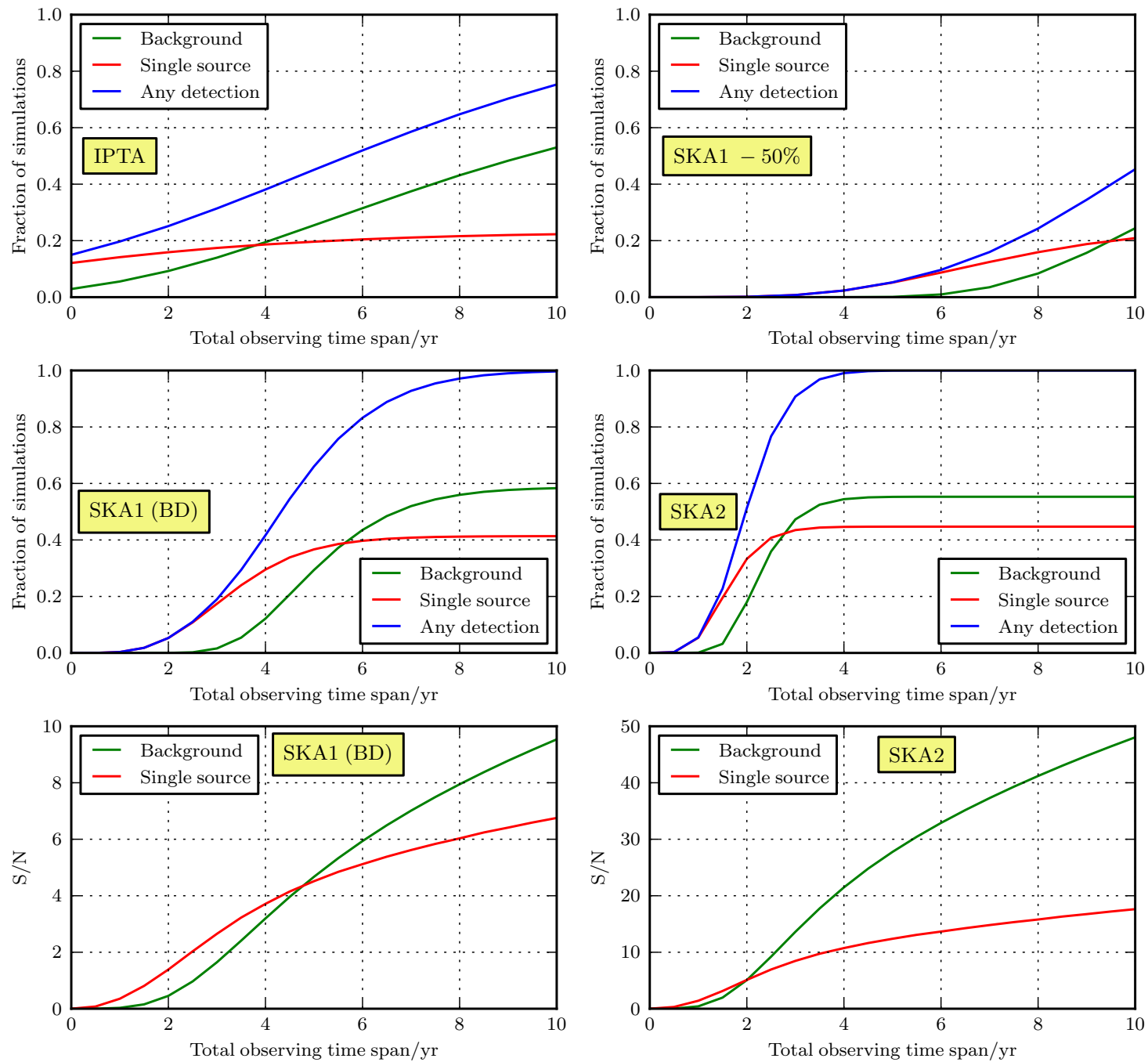

Figure 3: Detection probability for four different PTAs; top left: IPTA; top right: SKA1 at an early science $(50 \%)$ sensitivity level; middle left: SKA1 as defined in the baseline design document; middle right: SKA2. The top and middle panels show the predictions on the first GW detection by different PTAs as a function of time. In the IPTA case, the starting point corresponds to the present day, after 10 years of previous observations. In the SKA cases, the starting points correspond to the beginning of the SKA data-taking, disregarding previous data. The green curve gives the fraction of simulations of the Universe (which can be considered as a probability) in which a stochastic background is detected first. The red curve gives the probability of a single binary being detected first. The blue curve gives the overall probability of detecting any kind of GW signal. We assume that a GW signal is detected when it produces a signal-to-noise ratio (S/N) larger than a certain threshold. The chosen $\mathrm{S} / \mathrm{N}$ threshold is 4 , which may be considered realistic for single binaries, but a bit conservative for a background. A more detailed study can be found in Rosado, Sesana \& Gair (in prep.). The S/N of a detection of a single binary and of a background are obtained as described in Sesana \& Vecchio (2010) and Siemens et al. (2013), respectively, and are plotted as function of time in the bottom plots for SKA1 and SKA2 PTAs. 
or degraded receiver performance), if only a small frequency band was available or without the possibility of sub-arraying the telescope. The main effect of a reduced sensitivity on the SKA1 is a prolonged timescale for all GW detections. For example, in the case that the sensitivity of the SKA1 is reduced by $50 \%$, the overall GW detection probability after five years is reduced to $10 \%$.

\section{The future of gravitational wave astronomy}

SKA1, or the IPTA using pre-existing telescopes, is likely to make the first direct detection of GWs and will start to probe the properties of those waves. With the increased sensitivity of SKA2, gravitational wave astronomy will become a reality and the detailed properties of GWs can be studied. Unexplored territories of both astronomy and physics will be unveiled for the first time. The foreseeable breakthroughs include:

- Constraining models for galaxy evolution: Determining the GW properties (amplitude, spectral index, anisotropy or source distribution) of a GW background from coalescing supermassive black hole systems will lead to an improved understanding of galaxy evolution and black hole growth. With SKA2 it will eventually be possible to study the high end of the SMBHB mass distribution at low redshifts.

- Source localisation and population: By decomposing the stochastic GW background on a basis of spherical harmonics (Mingarelli et al. 2013), it is possible to characterise the GW background on any angular scale up to the maximum angular resolution of the PTA. This characterisation of the background can confirm its cosmological origin, but is also completely general. It is therefore possible to use this method to find GW hotspots, which may help to confine sky areas which need to be covered by continuous wave searches. Bayesian search techniques have been recently developed by Taylor \& Gair (2013), and are being implemented in anisotropic stochastic GW background searches. A CMB-like search has also been recently proposed by Gair et al. (2014), which can be used to recover the components of an arbitrary background.

- Testing gravity in the strong-field regime: Although there is still lack of a consistent framework to quantise the well accepted theory of general relativity, the quantisation for weak GWs was succesfully initialised in the 1960s. There are two basic intrinsic properties for particles: their mass and spin states. In general relativity, the corresponding particles, the gravitons, are zero-mass spin-2 particles. However, other theories of gravity predict up to six polarisation states of GWs. Investigating the polarisation states and constraining the mass of the graviton with a PTA (Lee et al. 2008, 2010; Cordes \& Shannon 2012; Chamberlin \& Siemens 2012; Lee 2013) will help to understand the intrinsic symmetry of the gravitational interaction and may give valuable inputs for quantising gravity in the strong-field regime, which is one of the key questions of modern theoretical physics. While timing of binary pulsars is providing important insights into the dynamics of gravitation with strongly-gravitating bodies (Shao et al. 2015), PTAs will present an unique opportunity to study gravitation in the radiative regime with GWs. 
- Studying and testing cosmological GWs: As shown by Sanidas et al. (2012), cosmic strings are predicted to generate a different GW background compared to a background generated by SMBHBs. The properties of a cosmic string-generated GW background are defined by their string linear energy density, the details of the string GW emission mechanism (kinks and cusps), the birth-scale of cosmic string loops, the intercommutation probability (the probability with which two cosmic string segments exchange partners when crossing each other) and possible deviations from the simple scaling evolution (e.g. Sousa \& Avelino 2013). By identifying the GW background generated by cosmic strings, we will get access to a unique cosmological lab that will give us access to physics at an energy scale close to the GUT scale. In the case of cosmic superstrings, the expected results are even more profound since we will achieve a measurement of a fundamental string theory parameter for the first time, such as the fundamental string coupling or the compactification/warping scale. By measuring their properties, we will be able to investigate underlying dynamics and make a distinction between cosmic strings and superstrings. Besides the cosmic string stochastic GWB, an SKA PTA project might also be able to detect a stochastic GWB created by several other mechanisms in the early Universe, (e.g. Fenu et al. 2009; Figueroa et al. 2013).

- GW distance scales: When a sub-lightyear accuracy for pulsar distances can be achieved with SKA2 (Lee et al. 2011), Galactic dynamics can be investigated. A PTA is a unique type of GW detector working in the short wavelength regime, i.e. many radiation wavelengths separate pulsar pairs from each other and the Earth. This makes the angular resolution of PTA higher ( $\delta \theta \simeq \lambda / D \sim 1$ arcmin; Lee et al. 2011) if we know the exact pulsar distance. With SKA1-MID, the pulsar distance will not be measured accurate enough, which prevents us from achieving such high resolution. Nevertheless, anisotropies in a GW background will still result in a different decomposition of the Hellings \& Downs curve. When the nature of the anisotropy can be measured (Taylor \& Gair 2013), the nature of the population of the GW sources can be studied (Mingarelli et al. 2013). With the SKA2, the pulsar timing parallax will bring the pulsar distance error down to a few light years. Such precise distance information will:

I) make the PTA signal to single $\mathrm{GW}$ sources coherent, allowing perform $\mathrm{GW}$ interferometric imaging of the whole sky with angular resolutions of arcminutes (Lee et al. 2011). This will enable the search for electromagnetic counterparts to the GW sources, possibly enabling the follow-ups of GW sources with the traditional astronomical tools which can lead to the study of galaxy mergers (Burke-Spolaor 2013; Rosado \& Sesana 2014; Deane et al. 2015).

II) allow us to measure the mass and spin of a SMBHB and map the non-linear dynamics of the gravitational field (Mingarelli et al. 2012); when the pulsar term can be distinguished from the Earth term, each pulsar term sees a slightly different piece of the evolutionary history of the binary since its orbital period evolves over the light travel time between the Earth and the pulsar. Concatenating these different snapshots of the waveform may allow for the measurement of the mass and spin of the binary, and furthermore, measuring postNewtonian parameters up to 1.5 order.

- Probing the inflationary era of the universe: There are two major methods to probe the inflationary era of the universe with PTA. The first one is to examine relic GWs, which give 
constraints on the tensor-to-scalar ratio (Zhao et al. 2013). The second one is to check the effects of structure formation, e.g. primordial black hole population, which give us information on the primordial density fluctuation (Saito \& Yokoyama 2009).

\section{Conclusions}

The first direct detection of GWs in the nano-Hertz regime using PTA observations is expected within a decade. The SKA will play an important role in this, either through making the first detection, or by confirming the detection independently, and providing the sensitivity to characterise continuous sources as well as the stochastic GW background. In general, pulsar timing precision will be greatly enhanced by the unprecedented collecting area and bandwidth of the SKA, and PTA sensitivity will be revolutionised though regular observations of a large number of pulsars. These would include many of the weaker pulsars wich may - through the added sensitivity of the array for the first time be timed at the precision that is required for PTA work.

In the early stages of the science, even before the SKA PTA achieves a direct GW detection, these data sets will already enable exciting scientific results; for example tests of theories of gravity, studies of binary and millisecond pulsars, searches for irregularities in the terrestrial time standards, and studies of the interstellar medium. While these results are scientifically interesting in their own right, they will also aid the characterisation of the pulsars and the observing system in a way that continuously enhances our sensitivity to GWs. Without a doubt, a PTA is an ideal experiment for the SKA.

\section{References}

Ade, P. A. R. et al. (Bicep2 Collaboration) 2014, Physical Review Letters, 112, 241101

Armstrong, J. W., Rickett, B. J., \& Spangler, S. R. 1995, ApJ, 443, 209

Arzoumanian, Z., Brazier, A., Burke-Spoloar, S., et al. 2014, ApJ, 794, 141

Babak, S. \& Sesana, A. 2012, Phys. Rev. D, 85, 044034

Bhat, N. D. R., Cordes, J. M., Camilo, F., et al. 2004, ApJ, 605, 759

Boyle, L. \& Pen, U.-L. 2012, Phys. Rev. D, 86, 124028

Brisken, W. F., Macquart, J.-P., Gao, et al. 2010, ApJ, 708, 232

Burke-Spolaor, S. 2013, Class. Quant Grav., 30, 224013

Chamberlin, S. J. \& Siemens, X. 2012, Phys. Rev. D, 85, 082001

Champion, D. J., Hobbs, G. B., Manchester, et al. 2010, ApJ, 720, L201

Cordes, J. M. \& Jenet, F. A. 2012, ApJ, 752, 54

Cordes, J. M., Kramer, M., Lazio, T. J. W., et al. 2004, New Astr., 48, 1413

Cordes, J. M. \& Shannon, R. M. 2012, ApJ, 750, 89

Deane, R., Paragi, Z., Jarvis, M., et al., 2015, "Multiple supermassive black hole systems: SKA's future leading role", in proc. Advancing Astrophysics with the Square Kilometre Array, PoS(AASKA14)151

Deller, A. T., Boyles, J., Lorimer, D. R., et al. 2013, ApJ, 770, 145

Demorest, P. B. 2011, MNRAS, 416, 2821

Demorest, P. B., Ferdman, R. D., Gonzalez, M. E., et al. 2013, ApJ, 762, 94 
Detweiler, S. 1979, ApJ, 234, 1100

eLisa Constortium: Seoane, P. A., Aoudia, S., et al. 2013, arXiv:1305.5720

Ellis, J. A. 2013, Class. Quant Grav., 30, 224004

Ellis, J. A., Siemens, X., \& Creighton, J. D. E. 2012, ApJ, 756, 175

Fenu, E., Figueroa, D. G., Durrer, R., et al. 2009, Journal of Cosm. and Astro-Particle Phys., 10, 5

Figueroa, D. G., Hindmarsh, M., \& Urrestilla, J. 2013, Phys. Rev. Lett., 110, 101302

Foster, R. S. \& Backer, D. C. 1990, ApJ, 361, 300

Gair, J. R., Romano, J. D., Taylor, S., \& Mingarelli, C. M. F. 2014, Phys. Rev. D, 90, 082001

Hassall, T. E., Stappers, B. W., Hessels, J. W. T., et al. 2012, A\&A, 543, A66

Hellings, R. W. \& Downs, G. S. 1983, ApJ, 265, L39

Hobbs, G., Coles, W., Manchester, R. N., et al. 2012, MNRAS, 427, 2780

Hobbs, G., Dai, S., Manchester, R. N., et al.2014, arXiv: 1407.0435

Hobbs, G., Lyne, A. G., \& Kramer, M. 2010, MNRAS, 402, 1027

Hobbs, G., Lyne, A. G., Kramer, M., et al. 2004, MNRAS, 353, 1311

Hobbs, G. B., Edwards, R. T., \& Manchester, R. N. 2006, MNRAS, 369, 655

Jenet, F. A., Hobbs, G. B., van Straten, W., et al. 2006, ApJ, 653, 1571

Jenet, F. A., Lommen, A., Larson, S. L., \& Wen, L. 2004, ApJ, 606, 799

Kaspi, V. M., Taylor, J. H., \& Ryba, M. 1994, ApJ, 428, 713

Keith, M. J., Coles, W., Shannon, R. M., et al. 2013, MNRAS, 429, 2161

Kramer, M., Backer, D. C., Cordes, J. M., et al. 2004, New Astron. Rev., 48, 993

Kramer, M. \& Champion, D. J. 2013, Class. Quant Grav., 30, 224009

Kramer, M. \& Stappers, B. 2010, in ISKAF2010 Science Meeting, arXiv: 1009.1938

Lee, K., Jenet, F. A., Price, R. H., et al. 2010, ApJ, 722, 1589

Lee, K. J. 2013, Class. Quant Grav., 30, 224016

Lee, K. J., Bassa, C. G., Janssen, G. H., et al. 2014, MNRAS, 441, 2831

Lee, K. J., Bassa, C. G., Janssen, G. H., et al. 2012, MNRAS, 423, 2642

Lee, K. J., Jenet, F. A., \& Price, R. H. 2008, ApJ, 685, 1304

Lee, K. J., Wex, N., Kramer, M., et al. 2011, MNRAS, 414, 3251

Liu, K., Desvignes, G., Cognard, I., et al. 2014, MNRAS, 443, 3752

Liu, K., Keane, E. F., Lee, K. J., et al. 2012, MNRAS, 420, 361

Lorimer, D. R. \& Kramer, M. 2005, Handbook of Pulsar Astronomy (Cambridge University Press)

Lyne, A., Hobbs, G., Kramer, M., et al. 2010, Science, 329, 408

Madison, D. R., Chatterjee, S., \& Cordes, J. M. 2013, ApJ, 777, 104

Manchester, R. N., Hobbs, G., Bailes, M., et al. 2013, PASA, 30, 17

Manchester, R. N. \& IPTA. 2013, Class. Quant Grav., 30, 224010

Mingarelli, C. M. F., Grover, K., Sidery, T., et al. 2012, Phys. Rev. Lett., 109, 081104

Mingarelli, C. M. F., Sidery, T., Mandel, I., \& Vecchio, A. 2013, Phys. Rev. D, 88, 062005

Ölmez, S., Mandic, V., \& Siemens, X. 2010, Phys. Rev. D, 81, 104028

Osłowski, S., van Straten, W., Demorest, P., \& Bailes, M. 2013, MNRAS, 430, 416

Osłowski, S., van Straten, W., Hobbs, G. B., et al. 2011, MNRAS, 418, 1258

Paragi, Z., Godfrey, L., Reynolds, C., et al. 2015, "Very Long Baseline Interferometry with the SKA", in proc. Advancing Astrophysics with the Square Kilometre Array, PoS(AASKA14)143 Pennucci, T. T., Demorest, P. B., \& Ransom, S. M. 2014, ApJ, 790, 93 
Petiteau, A., Babak, S., Sesana, A., \& de Araújo, M. 2013, Phys. Rev. D, 87, 064036

Phinney, E. S. 2001, arXiv:astro-ph/0108028

Pitkin, M., Reid, S., Rowan, S., \& Hough, J. 2011, Living Reviews in Relativity, 14, 5

Planck Collaboration XXX 2014, arXiv:1409.5738

Ravi, V., Wyithe, J. S. B., Hobbs, G., et al. 2012, ApJ, 761, 84

Ravi, V., Wyithe, J. S. B., Shannon, et al. 2014, MNRAS, 442, 56

Romani, R. W. 1989, in Timing Neutron Stars, ed. H. Ögelman \& E. P. J. van den Heuvel, 113-117

Rosado, P. A. \& Sesana, A. 2014, MNRAS, 439, 3986

Saito, R. \& Yokoyama, J. 2009, Phys. Rev. Lett., 102, 161101

Sanidas, S. A., Battye, R. A., \& Stappers, B. W. 2012, Phys. Rev. D, 85, 122003

Sanidas, S. A., Battye, R. A., \& Stappers, B. W. 2013, ApJ, 764, 108

Sesana, A. 2013a, Class. Quant Grav., 30, 224014

Sesana, A. 2013b, MNRAS, 433, L1

Sesana, A. \& Vecchio, A. 2010, Phys. Rev. D, 81, 104008

Sesana, A., Vecchio, A., \& Colacino, C. N. 2008, MNRAS, 390, 192

Shannon, R. M. \& Cordes, J. M. 2010, ApJ, 725, 1607

Shannon, R. M. \& Cordes, J. M. 2012, ApJ, 761, 64

Shannon, R. M., Ravi, V., Coles, W. A., et al. 2013, Science, 342, 334

Shao, L., Stairs, I. H., Antoniadis, J., et al. 2015, "Testing Gravity with Pulsars in the SKA Era", in proc. Advancing Astrophysics with the Square Kilometre Array PoS(AASKA14)042

Siemens, X., Ellis, J., Jenet, F., \& Romano, J. D. 2013, Class. Quant Grav., 30, 224015

Sousa, L. \& Avelino, P. P. 2013, Phys. Rev. D, 88, 023516

Stappers, B. W., Hessels, J. W. T., Alexov, A., et al. 2011, A\&A, 530, A80

Taylor, J. H. \& Weisberg, J. M. 1982, ApJ, 253, 908

Taylor, S. R. \& Gair, J. R. 2013, Phys. Rev. D, 88, 084001

Tong, M. L., Zhang, Y., Zhao, W., et al. 2014, Class. Quant Grav., 31, 035001

van Haarlem, M. P., Wise, M. W., Gunst, A. W., et al. 2013, A\&A, 556, A2

van Haasteren, R. \& Levin, Y. 2010, MNRAS, 401, 2372

van Haasteren, R., Levin, Y., Janssen, G. H., et al. 2011, MNRAS, 414, 3117

van Haasteren, R., Levin, Y., McDonald, P., \& Lu, T. 2009, MNRAS, 395, 1005

Verbiest, J. P. W., Bailes, M., Coles, W. A., et al. 2009, MNRAS, 400, 951

Walker, M. A., Demorest, P. B., \& van Straten, W. 2013, ApJ, 779, 99

Yardley, D. R. B., Coles, W. A., Hobbs, G. B., et al. 2011, MNRAS, 414, 1777

Yardley, D. R. B., Hobbs, G. B., Jenet, F. A., et al. 2010, MNRAS, 407, 669

Zhao, W., Zhang, Y., You, X.-P., \& Zhu, Z.-H. 2013, Phys. Rev. D, 87, 124012 